\section{Serotonin Release Assay}

\section{T. Stief und P. Kiefer}

Institut für Laboratoriumsmedizin und Pathobiochemie, Krankenhaus der Philipps-Universität, Marburg, Deutschland

\section{Synonym(e) SRA}

Englischer Begriff serotonin release assay

Definition SRA misst die Aktivierung von Thrombozyten durch Antikörper, die gegen einen Komplex von Heparin und Plättchenfaktor 4 gerichtet sind. Der SRA wird mit Spenderthrombozyten durchgeführt und gilt als Goldstandard der Heparin-induzierten Thrombozytopenie(HIT)-2-Testung. Der SRA ist wegen seiner Komplexität Speziallaboren vorbehalten.

Beschreibung Die Aktivierung der Plättchen durch Antikörper im Blut des Probanden ist der Freisetzung des $>$ Serotonin aus den Granula der Thrombozyten proportional. Das aus den Granula freigesetzte Serotonin wird durch einen $>$ Enzymimmunoassay (EIA-SRA) oder durch $>$ Hochleistungs-Flüssigkeitschromatographie (HPLC-SRA) bestimmt.
Der SRA gilt als $\triangleright$ Goldstandard zur Erfassung von HIT2-induzierenden Antikörpern. Sensitivität ( $\triangleright$ Sensitivität, diagnostische) und Spezifität ( $\triangleright$ Spezifität, diagnostische) werden mit $90 \%$ bzw. mit bis zu $100 \%$ angegeben. Häufig wird der Test auch eingesetzt, um schwache Ergebnisse des hochsensitiven Screeningtests (PF4/ELISA) zu bestätigen. HLA-Klasse-I-Autoantikörper oder Antikörper gegen Plättchen-spezifische Antigene können zu einer Heparin-unabhängigen Freisetzung von Serotonin führen. Bei allen HIT-2Messungen sollte auch die systemische Thrombinaktivität im Plasma ( $\alpha 2 \mathrm{M}-\mathrm{F} 2 \mathrm{a})$ gemessen werden, da Thrombin ein starker Thrombozytenaktivator ist.

Normbereich 19-62 ng/mL (jeweils 2,5-97,5 Perzentile; HIT-2-Patienten: $244-7987 \mathrm{ng} / \mathrm{ml})$.

\section{Literatur}

Harenberg J, Huhle G, Giese C, Wang LC, Feuring M, Song XH, Hoffmann U (2000) Determination of serotonin release from platelets by enzyme immunoassay in the diagnosis of heparin-induced thrombocytopenia. Br J Haematol 109:182-186 